\title{
COMEDICATION OF RABEPRAZOLE SODIUM CAUSES POTENTIAL DRUG-DRUG INTERACTION WITH DIABETIC DRUG LINAGLIPTIN: In-vitro AND In-silico APPROACHES
}

\author{
Md. Jamal Hossain ${ }^{1,2, *}$ (D), Md. Shamiul Islam, Saimon Shahriar ${ }^{1}$, Sherejad Sanam4, \\ Talha Bin Emran ${ }^{5}$ (i), Chand Sultana Khatun ${ }^{6}$, Md. Rabiul Islam ${ }^{7}$, \\ Saikat Mitra ${ }^{3}$, Kuldeep Dhama ${ }^{8, *}$ \\ ${ }^{1}$ Department of Pharmaceutical Chemistry, Faculty of Pharmacy, University of Dhaka, Dhaka 1000, Bangladesh \\ ${ }^{2}$ Department of Pharmacy, State University of Bangladesh, 77 Satmasjid Road, Dhanmondi, Dhaka 1205, Bangladesh \\ ${ }^{3}$ Department of Pharmacy, Faculty of Pharmacy, University of Dhaka, Dhaka 1000, Bangladesh \\ ${ }^{4}$ Department of Pharmacy, Stamford University Bangladesh, 51 Siddeswari Road, Ramna, Dhaka 1217, Bangladesh \\ ${ }^{5}$ Department of Pharmacy, BGC Trust University Bangladesh, Chittagong 4381, Bangladesh \\ ${ }^{6}$ Department of Clinical Pharmacy and Pharmacology, Faculty of Pharmacy, University of Dhaka, Dhaka 1000, Bangladesh \\ ${ }^{7}$ Department of Pharmacy, University of Asia Pacific, 74/A Green Road, Farmgate, Dhaka 1205, Bangladesh \\ ${ }^{8}$ Division of Pathology, ICAR-Indian Veterinary Research Institute, Bareilly, Izatnagar, Uttar Pradesh 243122, India \\ Received - June 16, 2021; Revision - August 07, 2021; Accepted - August 25, 2021 \\ Available Online -August 30, 2021 \\ DOI: http://dx.doi.org/10.18006/2021.9(4).528.542
}

\section{KEYWORDS}

Bovine serum albumin

Molecular docking

Multidrug therapy

Synchronous fluorescence spectroscopy

Thermogravimetric analysis

\begin{abstract}
Drug-drug interaction is a notable concern among physicians when prescribing multi-therapy to the patients as concomitant administration of multi-drugs might cause unexpected adverse drug reactions. The main objective of this research is to predict a potential drug-drug interaction between two frequently used drugs by diabetic patients, an antidiabetic drug (linagliptin) and a proton pump inhibitor (rabeprazole sodium). Here, several in vitro techniques, including thermal (melting point, thermogravimetric analysis [TGA]), morphological (scanning electron microscopy [SEM] and X-ray powder diffraction [XRPD] analysis), highly sophisticated synchronous fluorescence, and in silico methods were applied to anticipate the potential drug-drug interaction between these stated drugs quickly. The melting point and TGA study revealed thermochemical properties, thermal stability profiles, and degradation patterns upon temperature rising of the formed complex and these precursor drugs. The SEM and XRPD have provided the morphological changes like particle shape and size distribution of the desired molecule that might be caused due to the potential drug-drug interactions.
\end{abstract}

* Corresponding author

E-mail: jamal.du.p48@gmail.com; jamalhossain@sub.edu.bd (Md. Jamal Hossain); kdhama@rediffmail.com (Dr. Kuldeep Dhama)

Peer review under responsibility of Journal of Experimental Biology and Agricultural Sciences.

Production and Hosting by Horizon Publisher India [HPI] (http://www.horizonpublisherindia.in/).

All rights reserved.
All the articles published by Journal of Experimental Biology and Agricultural Sciences are licensed under a Creative Commons Attribution-NonCommercial 4.0 International License Based on a work at www.jebas.org.

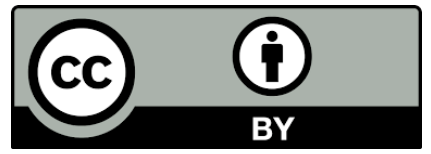


Besides, the drastic reduction of the quenching rate constant of linagliptin during interaction with bovine serum albumin in synchronous fluorescence also endorsed the potential drug-drug interaction. Furthermore, the drug-receptor docking analysis demonstrated that the binding affinity of the precursor ligands might be reduced due to the predicted drug-drug interaction. However, the current evidence warrants extensive investigation to confirm the above-stated potential drug-drug interaction in the larger animal model. Finally, clinical data need to be closely monitored during the treatment of diabetic patients prescribed with linagliptin and rabeprazole sodium.

\section{Introduction}

Polypharmacy has become a widespread treatment pattern during prescription for in-patients or out-patients in present days. As patients are experienced by multi pathological conditions, physicians must prescribe multidrug therapy. In some cases, evidence has been also reported that the use of combination therapies is superior to monotherapies, as they cure swiftly by acting as multiple receptor inhibitors (Weinstein et al., 2018). Similarly, drug interaction has taken place most concerning issue for a prescriber as well as a drug designer due to the effect of pharmacogenetic polymorphisms during concomitant therapy (Tannenbaum \& Sheehan, 2014). Drug-drug interaction analysis, which reports adverse drug events experienced by patients like discomfort or clinically significant harmfulness, has been a robust conception to pick out the best model of multidrug therapies and also predict the drug efficacy as well as the relationship of cellular components with drug mechanism of actions (Patel et al., 2017; Weinstein \& Zaman, 2017).

Drug interaction has recently turned into one of the most concerning issues for a physician during the prescription of multidrug therapy. It is vital to assess the potential interaction of a drug molecule during co-administration with another drug to study its efficacy and toxicological patterns. Various isoenzymes of cytochrome $\mathrm{P} 450$ and some drug transporters may be studied clinically for a prolonged time to predict potential drug-drug or drug-transporter interactions.

Linagliptin, chemically known as $(R)-8$-(3-aminopiperidin-1-yl)-7(but-2-ynyl)-3-methyl-1-((4-methylquinazolin-2-yl)methyl)-1 $H$ purine-2,6(3H,7H)-dione, is an orally administered selective dipeptidyl peptidase-IV (DPP-IV) inhibitor intended to be used in type 2 diabetes mellitus. It exhibited potent hypoglycemic activity with an advantage for patients with renal, liver, and cardiovascular complications (Abbas Moussa et al., 2019; Rosenstock et al., 2019). The drug shows its efficacy by binding with the enzyme DPP-IV competitively and reversibly, resulting in the inhibition of the degradation of the glucose-dependent insulinotropic polypeptide (GIP) and glucagon-like peptide-1 (GLP-1). As a result, these endogenous incretin hormones can remain in the circulation for a longer duration and reduce the blood glucose level in patients (Rajbangshi et al., 2018; Abbas Moussa et al., 2019; Arab et al., 2021). Linagliptin follows a non-linear pharmacokinetic profile with concentration-dependent binding with albumin and has a long half-life (more than 100 hours) and takes approximately 2 hours to reach maximum plasma concentration after being administered orally as a $5 \mathrm{mg}$ dose tablet. It shows minor first-pass metabolism; almost $90 \%$ of the drug is excreted in the unchanged form through the biliary and urinary systems (Ceriello \& Inagaki, 2017; Hossain et al., 2020c; Kang et al., 2020; Hossain et al., 2021).

Rabeprazole or its sodium salt belongs to a group named proton pump inhibitor and is intended to be used in peptic ulcer, gastroesophageal reflux disease (GERD), Zollinger-Ellison syndrome, and other acid-induced gastrointestinal disorders (Dash et al., 2018). It is chemically a substituted benzimidazole named 2(((4-(3-methoxypropoxy)-3-methylpyridin-2-yl)methyl)sulfinyl)-

$1 H$-benzo[d]imidazole (Sanad \& Challan, 2017). It is administered as enteric-coated tablets or capsules due to its acid-labile nature, and the drug is absorbed after passing the stomach. It is a prodrug that is activated by a strongly acidic environment, and then it selectively and irreversibly inhibits the $\mathrm{H}^{+} / \mathrm{K}^{+}$-ATPase enzyme (commonly known as the proton pump) situated at the inner secretory surface of the parietal cells of the stomach and thus reduces the basal and stimulated gastric acid secretion (Patel et al., 2007; Dash et al., 2018; Hossain et al., 2020c). Rabeprazole follows a simple pharmacokinetic model where it is metabolized extensively in the liver and mainly excreted through the kidney. It shows extensive protein binding and reaches the maximum plasma concentration within approximately 3.5 hours following the administration of a single dose (Hossain et al., 2020c). This drug displays several serious interactions with other pharmaceuticals, e.g. it enhances digoxin concentration and reduces ketoconazole levels in the plasma (Sanad \& Challan, 2017; Ochoa et al., 2020).

It is very well-known to all that diabetic patients may be potential consumers of proton pump inhibitors due to gastritis. This article provides some rapid and simple studies to anticipate the potential interactions that may be useful for the prevention of possible drugdrug interactions. The objective of this current research was to predict the plausible drug-drug interaction swiftly by several thermal, morphological, synchronous fluorescence spectroscopic, 
and computational analyses between linagliptin (LG) and rabeprazole sodium (RS). The interactions between these drugs have been studied by monitoring several in-vitro (melting point, TGA, SEM, XRPD, and synchronous fluorescence spectroscopy) and in-silico computational docking methods.

\section{Materials and Methods}

\subsection{Chemicals}

Linagliptin (LG), a dipeptidyl peptidase-4 (DPP-4) inhibitor (potency: 99.44\%), and rabeprazole sodium (RS) (potency: 96.65\%), a selective and irreversible proton pump inhibitor (PPI), were collected from ACI pharmaceutical Ltd., Dhaka, Bangladesh. All other chemicals such as potassium dihydrogen orthophosphate $\left(\mathrm{KH}_{2} \mathrm{PO}_{4}\right)$, potassium phosphate dibasic $\left(\mathrm{K}_{2} \mathrm{HPO}_{4}\right)$, phosphoric acid $85 \%$, bovine serum albumin (BSA), acetonitrile, methanol, ethanol, etc., for this research were analytical grade.

\subsection{Instruments and software}

A digitalized melting-point machine (WRS-1B), an electronic balance (AS 220.R2 Shimadzu, Japan), sonicator (UltrasonsMedili), pH meter (Orion Star A111), thermostatic water bath (Unitronic Orbital, P Spectra, Spain), TGA 50H (Shimadzu, Japan), JEOL/EO-JSM-6490 equipment, RIGAKU Ultima IV Xray Diffractometer, and so on, were utilized for the in-vitro analysis of drug-drug interaction. On the other hand, the F-7000 Spectrophotometer (Hitachi, Japan) was used for the synchronous fluorescence spectroscopic analysis, and various extensive computer-based software like PyRx, PyMol, Discovery Studio 4.5, etc., were run for molecular docking intended for receptor-based analysis.

\subsection{Preparation of pH 7.4 buffer}

To prepare $\mathrm{pH} 1.4$ phosphate buffer, $250 \mathrm{~mL}$ of $0.1 \mathrm{M} \mathrm{KH}_{2} \mathrm{PO}_{4}$ was added with $250 \mathrm{~mL}$ of $0.2 \mathrm{M} \mathrm{H}_{3} \mathrm{PO}_{4}$. Then the mixture was diluted with demineralized (DM) water to $1000 \mathrm{~mL}$. Finally, few drops of $85 \%$ phosphoric acid were added to adjust the $\mathrm{pH} 1.4$ (Hossain et al. 2020b).

\subsection{Synthesis and characterization of drug-drug complexation}

The drug-drug complex of LG-RS was designed, formed, and characterized by various in-vitro (chromatographic and spectroscopic) and in vivo methods disclosed by previous workers (Hossain et al., 2020c). Herein, we briefly reported the thermal, morphological, synchronous fluorescence quenching property, and molecular docking results due to the strong drug-drug interaction between LG and RS.

\subsection{Melting points}

A digital melting point apparatus was used to measure the melting point of both pure drugs and their 1:1 formed complex at atmospheric pressure.

\subsection{Thermogravimetric analysis (TGA)}

A TGA 50H (Shimadzu, Japan) was used to observe the degradation pattern of the pure drugs as well as the formed complex in respect of time and temperature rising. An aluminium pan was utilized to take thermograms up to $800^{\circ} \mathrm{C}$. The nitrogen gas was flowing at $10 \mathrm{~mL} / \mathrm{min}$ rate, and the temperature was rising at $10^{\circ} \mathrm{C} / \mathrm{min}$.

\subsection{Scanning electron microscope (SEM)}

The scanning electron microscope (SEM) images of the pure drugs LG and its formed complex with rabeprazole sodium were recorded by JEOL/EO-JSM-6490 equipment at accelerated voltage $10 \mathrm{kV}$. All the samples were coated with platinum layers for the analysis, and then images were taken in the most common mode of detection.

\subsection{X-ray powder diffraction (XRPD)}

The crystal structure is a vital parameter of a drug's morphological property, and measurement of the mean crystallite size of a drug is the precondition to explain its material characteristics. In the current study, Scherrer's formula was applied to enumerate the mean crystallite size of LG, RS, and LG-RS complex molecules by utilizing all the required parameters obtained from X-ray powder diffraction (XRPD) analysis (Ullah et al., 2013).

Scherrer's formula $\mathrm{D}=\frac{\mathrm{k} \lambda}{\beta \cos \theta}$

Where

D - crystallite size;

$\lambda$ - wavelength of incident of X-ray;

$\beta$ - Full width half maximum (FWHM) in radians;

$\theta$ - Bragg angle;

$\mathrm{k}$ - Shape factor (0.94)

The XRPD analyses for all three samples (LG, RS, and 1:1 formed complex) were carried out using RIGAKU Ultima IV X-ray Diffractometer at room temperature in the $2 \theta$ range of $20^{\circ}$ to $70^{\circ}$. Here, all the detailed conditions are stated as mentioned in Table 1. 
Table 1 Proper conditions during running XRPD for three samples of linagliptin, rabeprazole sodium, and their 1:1 formed complex.

\begin{tabular}{|cccc|}
\hline X-Ray & $40 \mathrm{kV}, 40 \mathrm{~mA}$ & Scan speed & $5.0000 \mathrm{deg} . / \mathrm{min}$. \\
\hline Goniometer & & Step width & $0.0200 \mathrm{deg}$. \\
\hline Attachment & - & Scan axis & 2 theta/theta \\
\hline Filter & & Scan range & $10.0000-70.0000 \mathrm{deg}$. \\
\hline CBO selection slit & - & Incident slit & $2 / 3$ deg. \\
\hline Diffracted beam mono. & Fixed Monochro. (U4) & Length limiting slit & - \\
\hline Detector & Scintillation counter & Receiving slit \#1 & $2 / 3$ deg. \\
\hline Scan mode & CONTINUOUS & Receiving slit \#2 & $0.3 \mathrm{~mm}$ \\
\hline
\end{tabular}

\subsection{Synchronous fluorescence quenching}

A highly sophisticated F-7000 spectrophotometer collected from Japan (Hitachi) was utilized for taking the synchronous fluorescence spectra of the targeted drugs and their formed 1:1 complex during the interaction with bovine serum albumin (BSA). The spectrophotometer was well-equipped with a $10 \mathrm{~mm}$ quartz cell. The concentrations of the aqueous samples of the ligands were varied from 1 to $10 \mathrm{mM}$ at physiological $\mathrm{pH} 7.4$, which was simulated by utilizing the freshly prepared phosphate buffer solution. In contrast, the strength of BSA was constant at $0.025 \%(\mathrm{w} / \mathrm{v})$ prepared by using the same buffer solution. To investigate the spectral characteristics of the tryptophan (Trp) and tyrosine (Tyr) of the serum protein, the difference between emission and excitation wavelengths $\left(\Delta \lambda=\lambda_{\mathrm{em}}-\lambda_{\mathrm{ex}}\right)$ were set at $15 \mathrm{~nm}$ and $60 \mathrm{~nm}$, respectively, during the scanning process of the mixed samples with BSA in the fluorescence spectrophotometer (Zhang et al., 2017). The temperature was controlled at $300 \mathrm{~K}$ during the whole process of the sample running in the fluorescence spectrophotometer. All the prepared samples were run repeatedly three times to keep the experimental error below $1 \%$, and the data obtained from the experiment might be reproducible.

A combination of the amino acids called tryptophan (Trp), tyrosine (Tyr), and phenylalanine (Phe) built the BSA protein, and mainly, these amino acids are primarily responsible for the intrinsic fluorescence characteristics. The inherent fluorescence properties of the biomolecules also vary, either quenching or dequenching, when interacting with any ligand or drug (Suryawanshi et al., 2016). Molecular interaction between drug and protein might cause the quenching of the fluorescence strength of the fluorophore ( $\mathrm{Li}$ et al., 2016). However, the current study is looking forward to investigating the stern-Volmer quenching constant and biomolecular quenching constant by utilizing the following relationship (Wang et al., 2019):

$$
\mathrm{F}_{\mathrm{o}} / \mathrm{F}=1+\mathrm{k}_{\mathrm{q}} \tau_{\mathrm{o}}[\mathrm{Q}]=1+\mathrm{K}_{\mathrm{SV}}[\mathrm{Q}]
$$

Where $F_{0}$ and $F$ mean the fluorescence intensity of the pure BSA and when the ligand is reacted with BSA, respectively. [Q] might be defined as the concentration of drug particles in the solution $(\mathrm{mol} / \mathrm{L}) . \mathrm{K}_{\mathrm{sv}}$ and $\mathrm{k}_{\mathrm{q}}$ are denoting the stern-Volmer quenching constant and biomolecular quenching rate constant, respectively. By utilizing the above-stated equation, a linear line was constructed by $\mathrm{F}_{0} / \mathrm{F}$ vs. [Q], and $\mathrm{K}_{\mathrm{Sv}}$ was determined from the value of the slope. $\tau 0$ has been defined as the average lifetime of the unreacted BSA, which was extrapolated from the previous literature, $\tau_{0}=10^{-8} \mathrm{~s}$ (Suryawanshi et al., 2016). The following formula was applied to measure the value of $\mathrm{k}_{\mathrm{q}}$ by utilizing the value of $\mathrm{K}_{\mathrm{sv}}$.

$$
\mathrm{k}_{\mathrm{q}}=\frac{\mathrm{K}_{\mathrm{sv}}}{\tau_{\mathrm{o}}}
$$

\subsection{Molecular docking}

\subsubsection{Preparation of protein}

The basic information regarding the preparation of dipeptidyl peptidase IV (DPP IV) and proton pump proteins were taken from Uniprot (https://www.uniprot.org/). The three-dimensional crystal structures of DPP IV in complex with inhibitor PZF (PDB ID: 2QJR) and proton pump (PDB ID: 4HQJ) were retrieved in .pdb format from the protein data bank (https://www.rcsb.org/). DPP IV in complex with inhibitor PZF was loaded to the PyMoL software package. The water molecules and the heteroatoms were selected and removed from the sequence of the protein. Water molecules and heteroatoms free protein were saved as .pdb format. This .pdb file was then opened to Swiss PDB Viewer, which was subsequently subjected to energy minimization (spdbv) using the steepest descent and conjugate gradient method to eliminate bad contacts of protein atoms. By following the same procedure, water molecules and heteroatoms from the proton pump were removed by PyMoL software packages, and energy was minimized by the Swiss PDB Viewer. The SDF format of both ligands was downloaded from the PubChem website (https://pubchem.ncbi.nlm.nih.gov/). 


\subsubsection{Ligand optimization and docking}

LG and RS were retrieved from PubChem. LG was imported to PyRx. The mmff94 force field was utilized with the steepest descent optimization algorithm, and a total of 2000 minimization steps were adopted for the energy minimization of LG. Then it was converted as an Autodock ligand for docking. The cleaned and minimized DPPIV was loaded to PyRx and converted as a macromolecule. After that, the ligand and the macromolecule were selected, and the grid box was generated according to the active site of the proteins by using Toggle Selection Spheres. For DPP IV; the centre was $\mathrm{X}: 9.55, \mathrm{Y}: 4.94$, and $\mathrm{Z}: 36.74$, and the dimensions were $41.3025,61.2067$, and 24.4269 , respectively for $\mathrm{X}, \mathrm{Y}$, and Z. After completing the docking, the result was collected in excel sheet, and the docking file was imported for further analysis. Again LG was imported to PyRx and optimized by using mmff94 force field with the steepest descent optimizing algorithm and a total of 2000 minimization steps and converted to AutoDock ligand. DPP IV combined with RS was loaded to PyRx and converted as a macromolecule. The ligand and the macromolecule were selected, and a grid box was generated according to the active site of the proteins. After completing the docking, the result was collected, and the docking file was imported. By following the similar procedure, RS was docked with Proton Pump, and then RS was docked with LG-bound PP where the grid box size was following: the centre was X:-9.87, Y: 92.26 , and Z: 66.51 , and the dimensions were $41.2766,28.2882$, and 44.4220 , respectively for $\mathrm{X}, \mathrm{Y}$, and Z-axis. The binding affinity was calculated as $\mathrm{kcal} / \mathrm{mole}$ as a unit for a negative score (Trott \& Olson, 2010; Hossain et al., 2020a). The ligands with the highest negative values of binding affinities indicate better binding affinities (Ahmed et al., 2021).

Finally, by using PyMoL Molecular Graphics System (version 1.7.4), ligand from the imported docking file and energy minimized protein was combined. Then Accelrys Discovery Studio 4.1 was used to analyze the lowest binding free energy conformer with the respective protein and to find out the possible binding interactions between the ligand and the protein.

\section{Results}

\subsection{Thermal analysis: meting point and TGA}

A digital melting point apparatus was used to measure the melting point of LG, RS, and their 1:1 formed complex at atmospheric

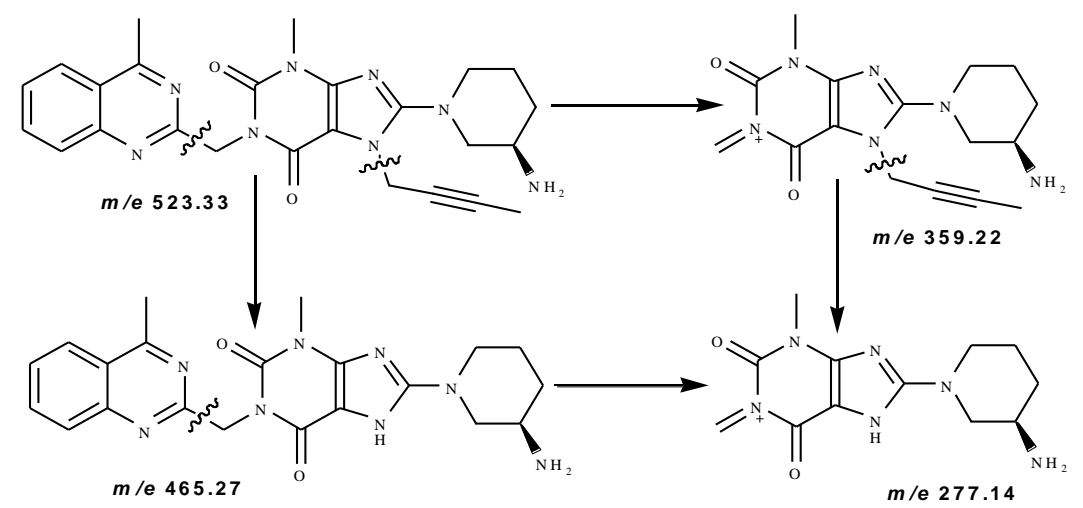

Scheme 1 Plausible degradation pathway of linagliptin at various in vivo conditions

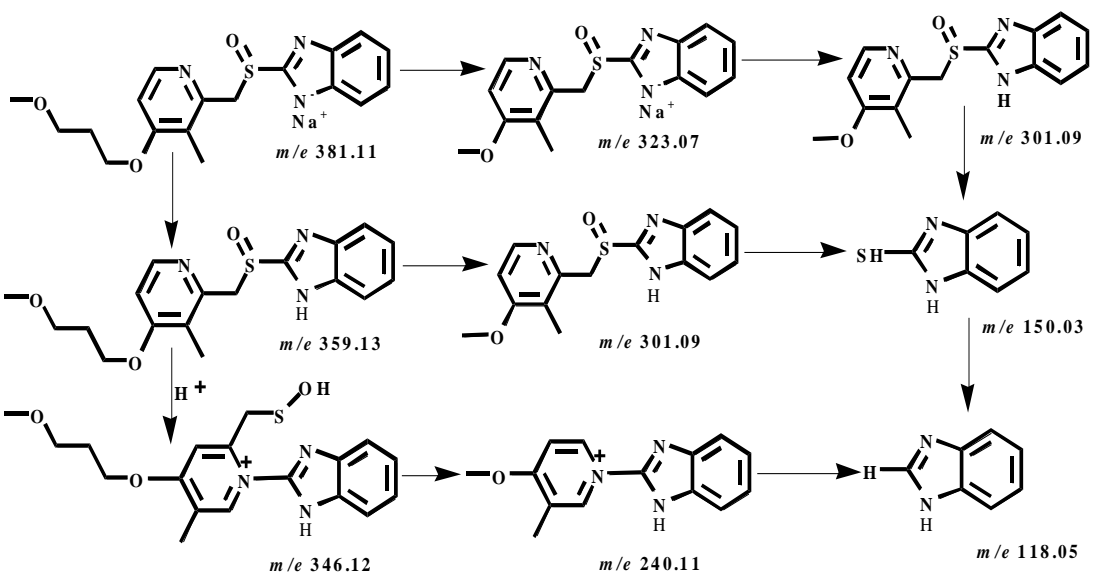

Scheme 2 Plausible degradation pathway of rabeprazole sodium at in vivo conditions

Journal of Experimental Biology and Agricultural Sciences http://www.jebas.org 
pressure. The melting point of the complex was quite different from its parent compounds. The melting point or decomposition temperature of the complex was $175-178^{\circ} \mathrm{C}$, whereas the pure LG and RS decomposed at $212^{\circ} \mathrm{C}$ and $88^{\circ} \mathrm{C}$, respectively (Figure 1A).

Thermograms of TGA of both pure drugs and their formed complexes were taken, and the overlaid TGA thermograms are displayed in figure 1B. The degradation patterns of both pure drugs and their complex concerning temperature rising are represented in table 2. From TGA data, it was confirmed that the complete burning of the formed complex occurred above $750^{\circ} \mathrm{C}$, which indicated its thermal stability. In TGA for pure linagliptin, $4 \%$ degradation from $0 \%$ was observed by increasing temperature from $21.90^{\circ} \mathrm{C}$ to $300.32^{\circ} \mathrm{C}$ which supports the degradation of pure linagliptin to its first moiety i.e. $\mathrm{m} / \mathrm{e} 523.20$ to 465.27 (Scheme 1). Similarly, all the degradation patterns and plausible fragmentation pathway of linagliptin were also stated in table 2 and scheme 2, supported by the TGA thermograms and confirmed from its previously published fragmentation pattern (Barden et al., 2017; Huang et al., 2018).

\subsection{Morphological analysis: color, XRPD and SEM}

\subsubsection{Color}

The color of LG was white, and the color of RS was white. However, the color of the solid LG-RS complex was deep brown.

\subsubsection{XRPD}

The X-ray diffractograms of LG, RS, and LG-RS complex are displayed in figure $2 \mathrm{~A}$. LG contained a crystalline structure which was exhibited through the number of peaks in the diffractogram. It showed 12 peaks ranging from 13.473 to $30.31^{\circ} 2 \theta$, which proves its crystalline structure. The intensity of these peaks was also stronger. RS showed only one peak, at $19.37^{\circ} 2 \theta$, meaning it was less crystalline than LG. The LG-RS complex also showed only one peak at $23.29^{\circ} 2 \theta$. However, its intensity was stronger than that of RS but less strong than those of LG. From these diffractograms, it was evident that RS reduced the crystalline characteristics of LG and the LG-RS complex displayed intermediate crystalline characteristics in comparison with LG and RS. The change in

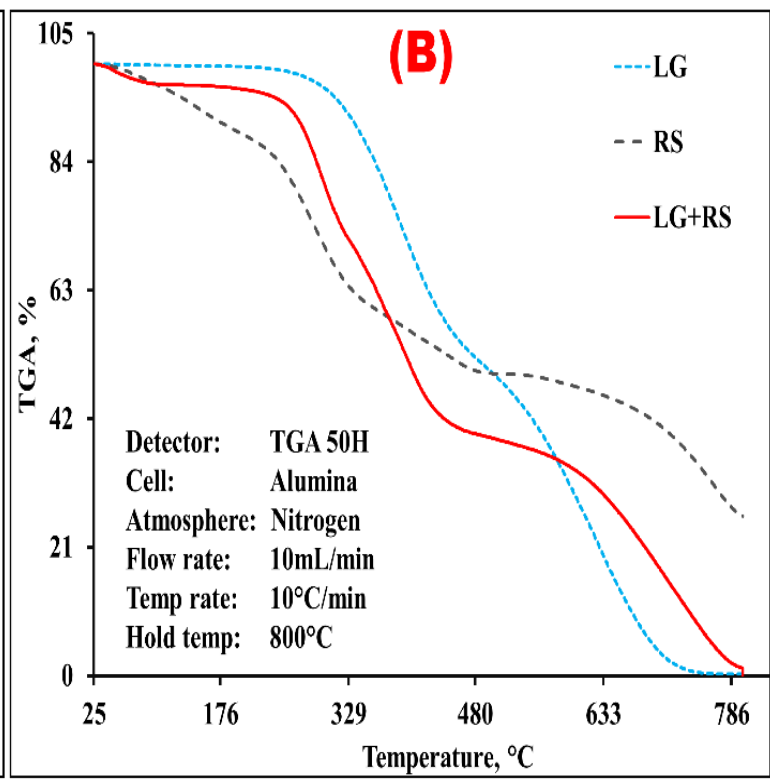

Figure 1 Thermal characteristics of linagliptin, rabeprazole sodium, and their 1:1 formed complex. (A) Graphical presentation of melting points of linagliptin, rabeprazole sodium, and their 1:1 complex. (B) Overlaid TGA curve of linagliptin, rabeprazole sodium, and their 1:1 formed complex. [TGA = Thermogravimetric analysis, $\mathrm{LG}=$ linagliptin and $\mathrm{RS}=$ rabeprazole sodium $]$.

Table 2 Degradation patterns of linagliptin (LG), rabeprazole sodium (RS) and their formed complex from TGA.

\begin{tabular}{|c|c|c|c|c|c|}
\hline Sample & \multicolumn{5}{|c|}{$\%$ degradation with temperature rising } \\
\hline LG & $\begin{array}{c}0 \% \\
\text { at } 21.90^{\circ} \mathrm{C}\end{array}$ & $\begin{array}{c}4 \% \\
\text { at } 300.311^{\circ} \mathrm{C}\end{array}$ & $\begin{array}{c}44.01 \% \\
\text { at } 453.11^{\circ} \mathrm{C}\end{array}$ & $\begin{array}{c}65.01 \% \\
\text { at } 578.38^{\circ} \mathrm{C}\end{array}$ & $\begin{array}{c}99.79 \% \\
\text { at } 799.43^{\circ} \mathrm{C}\end{array}$ \\
\hline RS & $\begin{array}{c}0 \% \\
\text { at } 24^{\circ} \mathrm{C}\end{array}$ & $\begin{array}{c}15.63 \% \\
\text { at } 242.78^{\circ} \mathrm{C}\end{array}$ & $\begin{array}{c}36.90 \% \\
\text { at } 332.92^{\circ} \mathrm{C}\end{array}$ & $\begin{array}{c}56.16 \% \\
\text { at } 667.17^{\circ} \mathrm{C}\end{array}$ & $\begin{array}{c}73.93 \% \\
\text { at } 799.48^{\circ} \mathrm{C}\end{array}$ \\
\hline LG-RS complex & $\begin{array}{c}0 \% \\
\text { at } 25.60^{\circ} \mathrm{C}\end{array}$ & $\begin{array}{c}6.91 \% \\
\text { at } 93.09^{\circ} \mathrm{C}\end{array}$ & $\begin{array}{c}56.88 \% \\
\text { at } 435.84^{\circ} \mathrm{C}\end{array}$ & $\begin{array}{c}66.47 \% \\
\text { at } 602.64^{\circ} \mathrm{C}\end{array}$ & $\begin{array}{c}98.49 \% \\
\text { at } 799.55^{\circ} \mathrm{C}\end{array}$ \\
\hline
\end{tabular}

Journal of Experimental Biology and Agricultural Sciences http://www.jebas.org 
Table 3 Measurement of mean crystallite size of linagliptin (LG), rabeprazole sodium (RS) and their 1:1 formed complex through utilizing the data obtained from XRPD analysis.

\begin{tabular}{|c|c|c|c|c|c|c|c|}
\hline \multicolumn{8}{|c|}{ Mean crystallite size measurement } \\
\hline $\begin{array}{l}\text { Compound } \\
\text { code }\end{array}$ & 2-theta (deg) & theta & theta (rad) & FWHM & FWHM (rad) & $\mathrm{D}(\mathrm{nm})$ & $\begin{array}{c}\text { Av. D }(\mathrm{nm}) \\
(\text { mean } \pm \mathrm{SD})\end{array}$ \\
\hline \multirow{12}{*}{ LG } & 13.473 & 6.7365 & 0.117574105 & 0.88 & 0.015358897 & 9.08682 & \\
\hline & 14.389 & 7.1945 & 0.125567713 & 0.42 & 0.007330383 & 19.05765 & \\
\hline & 15.439 & 7.7195 & 0.134730692 & 0.42 & 0.007330383 & 19.08052 & \\
\hline & 17.614 & 8.807 & 0.153711147 & 0.88 & 0.015358897 & 9.131752 & \\
\hline & 18.88 & 9.44 & 0.164759081 & 0.38 & 0.006632251 & 21.18477 & \\
\hline & 20.83 & 10.415 & 0.181776042 & 0.38 & 0.006632251 & 21.24796 & \\
\hline & 22.695 & 11.3475 & 0.198051237 & 0.32 & 0.005585054 & 25.31102 & $16.50 \pm 5.04$ \\
\hline & 23.91 & 11.955 & 0.208654112 & 0.57 & 0.009948377 & 14.2408 & \\
\hline & 26.463 & 13.2315 & 0.23093324 & 0.42 & 0.007330383 & 19.42323 & \\
\hline & 27.01 & 13.505 & 0.235706715 & 0.6 & 0.010471976 & 13.61169 & \\
\hline & 27.846 & 13.923 & 0.243002192 & 0.5 & 0.008726646 & 16.36314 & \\
\hline & 30.31 & 15.155 & 0.264504648 & 0.8 & 0.013962634 & 10.28415 & \\
\hline $\mathrm{RS}$ & 19.37 & 9.68 & 0.169046294 & 15 & 0.261799000 & 0.560938 & $0.560938 \pm 0$ \\
\hline LG-RS & 23.29 & 11.64 & 0.203156800 & 15 & 0.560938000 & 0.498148 & $0.498148 \pm 0$ \\
\hline
\end{tabular}

crystal structure and morphology is crucial because it may alter the pharmacological activity and cause new side effects (Chuah et al., 2014).

The mean crystalline sizes of these materials have been enumerated, and the results are displayed in table 3 . The average diameter of LG crystals was found to be $16.502 \mathrm{~nm}$, whereas the average diameter of RS crystals was only $0.561 \mathrm{~nm}$. For the LGRS complex, it was $0.498 \mathrm{~nm}$. The decrease in particle sizes might have happened due to the interaction of LG with RS. The complex formation process might have attributed to this issue as well. Another interesting finding was that the particle size of LG varied much more than the rest. Its diameter ranged from $9.087 \mathrm{~nm}$ to $25.311 \mathrm{~nm}$ resulting in a standard deviation of $30.54 \%$. The particle size of RS and the complex did not vary this much. It may be stated that the homogeneity of the RS particles reduced the heterogeneity of LG particles. The reduced particle size of the complex may cause aberrant pharmacological behavior in the patients as smaller particles have a higher surface area and show more absorption (Shariare et al., 2011).

\subsubsection{SEM}

The SEM photographs of LG and the LG-RS complex are displayed in figure $2 \mathrm{~B}$ and figure $2 \mathrm{C}$, respectively. These micrographs displayed the topographical changes followed by complexation. LG crystals appeared as irregular shaped and of different sizes. Their surface structure looked rough and with grooves. On the other hand, the surface of the complex looked

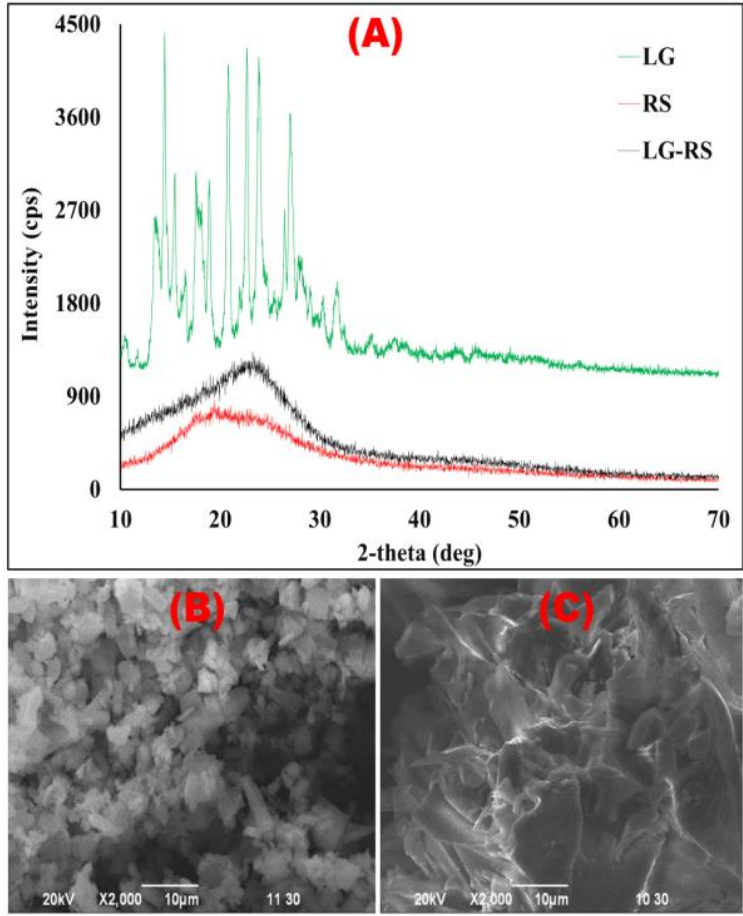

Figure 2 Morphological characteristics of linagliptin, rabeprazole sodium, and their 1:1 formed complex. (A) Comparative X-ray diffractograms of linagliptin, rabeprazole sodium, and their 1:1 complex. (B) SEM photograph of linagliptin. (C) SEM photograph of linagliptin during complexation with rabeprazole sodium [SEM $=$ Scanning electron microscopy, $\mathrm{LG}=$ linagliptin and $\mathrm{RS}=$ rabeprazole sodium $]$ 
comparatively smoother as well as of more homogenous sizes. The shape of the complex molecules was still irregular. The change in the size distribution might result from the complexation with RS, as RS molecules showed more homogenous size distribution in the XRPD analysis. Similar incidents have been observed by Ambike et al. (2005). It was also reported that RS particle size was much smaller than the LG one. It might attribute to the comparative smoothness of the complex, as smaller RS particles might have filled up the grooves of the rough LG particles.

\subsection{Synchronous fluorescence spectroscopic analysis}

The synchronous fluorescence spectra of ligand-added BSA solutions $(0.025 \% \mathrm{w} / \mathrm{v})$ are displayed in figure 3 . BSA solution alone showed high fluorescence intensity for both Tyr and Trp residues ( $\Delta \lambda$ values of $15 \mathrm{~nm}$ and $60 \mathrm{~nm}$, respectively), but that intensity was reduced upon the addition of various concentrations of the ligands (LG, RS, and LG-RS complex). LG ligand reduced the intensity for both residues. However, this degree of reduction was higher in the case of the Tyr residue. On the other hand, RS reduced the intensity for the Trp residue only. As a result, the LG-RS complex was found to cause the reduction of the intensity for the Trp residue, not for the Tyr residue. Besides, a higher reduction of the intensity indicates stronger quenching by the ligands through more stable ligand-protein binding. Therefore, it can be said that LG alone could bind to the BSA strongly through both Tyr and Trp residues, but the LG-RS complex could not interact with the Tyr residue to that degree, although interaction with the Trp residue was not hampered much. The reason for this change was the complexation with RS, as RS showed similar weak interaction with the Tyr residue. Similar results are also seen in the Stern-Volmer plots of these ligands (Figure 4). The graph for LG was not altered much in the case of the Trp residue, but it was completely changed in the case of the Tyr residue. It had to have happened due to the interaction of RS with the Tyr, and not the Trp, residue. The Stern-Volmer quenching constants and the quenching rate constants are calculated and shown in table 4 .

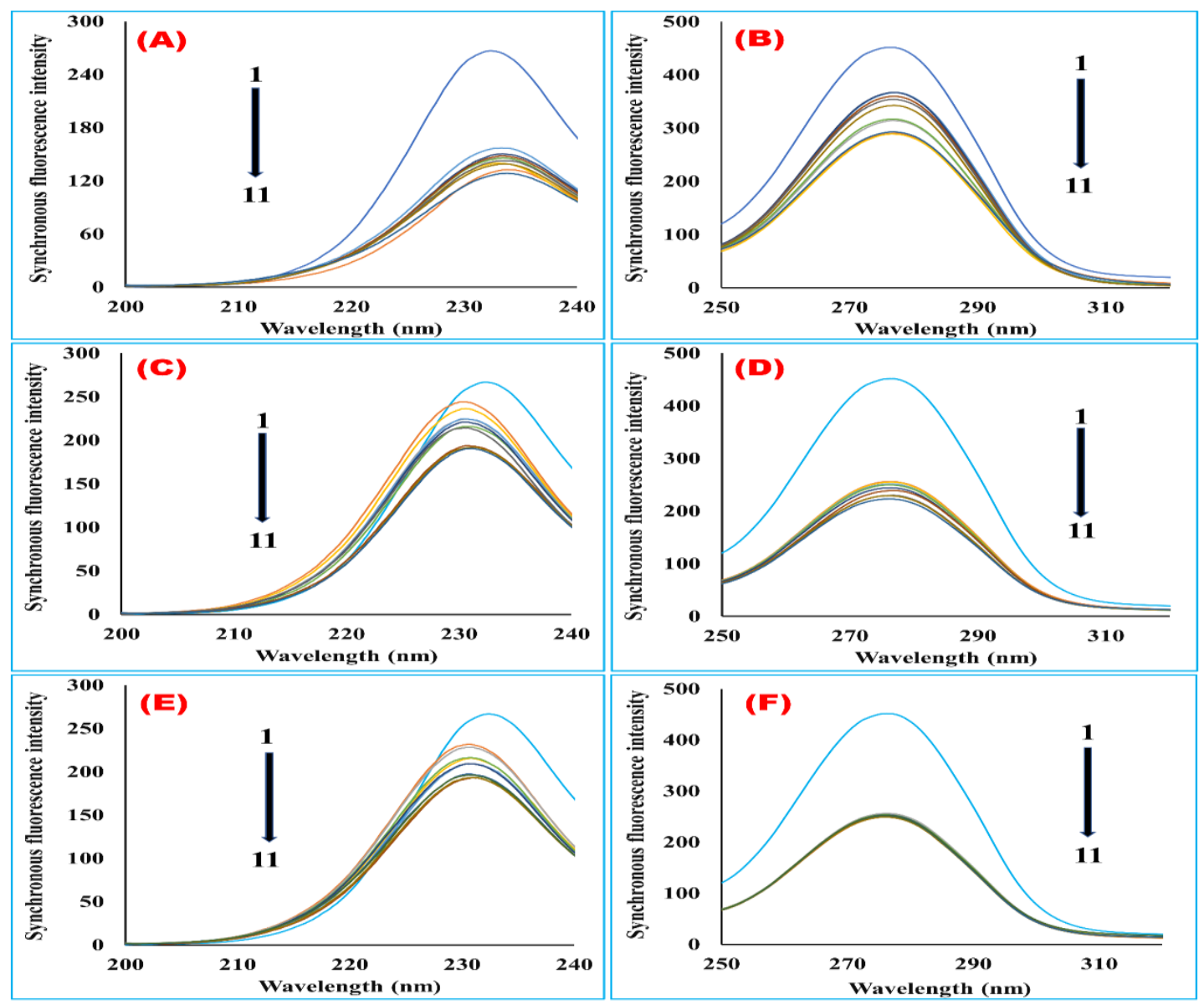

Figure 3 Synchronous fluorescence spectra of $0.025 \%$ (w/v) BSA solutions upon addition of ligands. Curves 1 to 11 indicated the spectra where the concentrations $(0,1,2,3,4,5,6,7,8,9,10 \mathrm{mM}$, respectively) of linagliptin (A, B), rabeprazole sodium (C, D), and their 1:1 formed complex (E, F). (A, C, E were for $\Delta \lambda=15 \mathrm{~nm}$ and B, D, F were for $\Delta \lambda=60 \mathrm{~nm}$. 

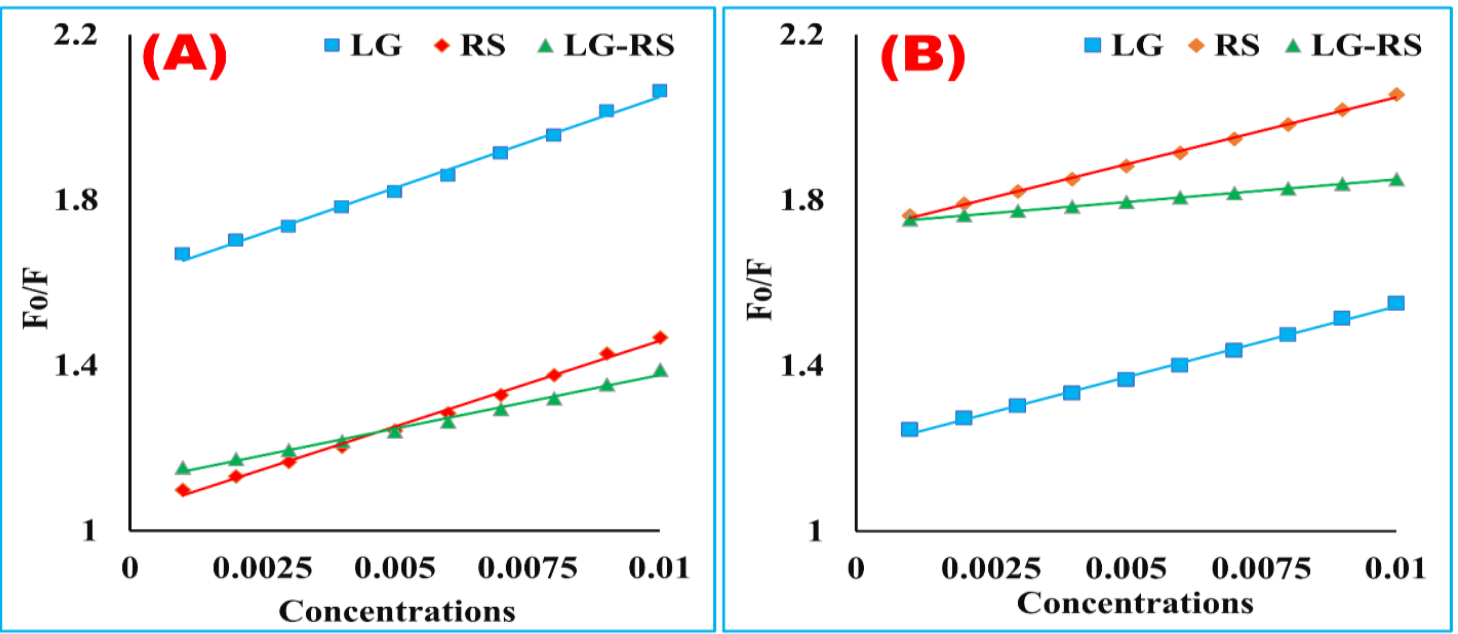

Figure 4 Synchronous fluorescence quenching of serum protein: Stern-Volmer plots with increased concentrations of linagliptin, rabeprazole sodium, and their 1:1 formed complex at $300 \mathrm{~K}$. (A) $15 \mathrm{~nm}$ and (B) $\Delta \lambda=60 \mathrm{~nm}$.[Here, LG = Linagliptin, RS = Rabeprazole sodium]

Table 4 Stern-Volmer quenching constants of the linagliptin-albumin, rabeprazole sodium-albumin, and 1:1 drug-drug complex-albumin systems during synchronous fluorescence quenching.

\begin{tabular}{|ccccc|}
\hline System & $\Delta \lambda(\mathrm{nm})$ & $\mathrm{K}_{\mathrm{sv}}\left(\mathrm{L} \cdot \mathrm{mole}^{-1}\right)$ & $\mathrm{k}_{\mathrm{q}}\left(\times 10^{10} \mathrm{~L} \cdot \mathrm{mole}^{-1} \cdot \mathrm{s}^{-1}\right)$ & $\mathrm{r}^{2}$ \\
\hline \multirow{2}{*}{ LG-Albumin } & 15 & 44.01 & 0.4401 & 0.993 \\
\cline { 2 - 5 } & 60 & 34.15 & 0.3415 & 0.997 \\
\hline \multirow{2}{*}{ RS- Albumin } & 15 & 41.57 & 0.4157 & 0.995 \\
\cline { 2 - 5 } & 60 & 32.44 & 0.3244 & 0.998 \\
\hline \multirow{2}{*}{ LG-RS- Albumin } & 15 & 25.92 & 0.2592 & 0.991 \\
& 60 & 10.88 & 0.1088 & 0.999 \\
\hline
\end{tabular}

Note: Here, LG = Linagliptin, RS = Rabeprazole sodium.

\subsection{Molecular docking analysis}

The binding properties of the LG with DPP-IV and RS with PP are investigated by the docking simulation of AutoDockVina (Figure 5). The outcome of docking analysis showed that LG has a binding affinity of $-9.6 \mathrm{kcal} / \mathrm{mol}$ with DPP-IV, whereas LG showed a binding affinity of $-8.0 \mathrm{kcal} / \mathrm{mol}$ when RS is attached to the binding pocket of DPP-IV (Figure 6 and Table 5). RS has a binding affinity of $-6.5 \mathrm{kcal} / \mathrm{mol}$ with $\mathrm{PP}$, but RS has a binding affinity of -6.3 $\mathrm{kcal} / \mathrm{mol}$ with LG attached PP. Various kinds of non-covalent interactions such as hydrogen bond, electrostatic bond, and hydrophobic interactions are found between LG and DPP-IV, LG and RS bound DPP-IV, RS and PP, RS and LG bound PP when the poses are predicted with AutoDock Vina (Figure 5 and Table 5). Besides, figure 6 indicates the highest binding affinity at the null RMSD (root mean square deviation), which postulates the best docking prediction. LG formed two hydrogen bonds with SER630 of DPP-IV, and one intra-hydrogen bond is formed. There are six hydrophobic bonds between LG and DPP-IV in which TYR547, TRP629, TYR662, TYR666 amino acids are involved. These three hydrogen bonds and six hydrophobic bonds help to stabilize the LG- DPP-IV complex. When LG is docked against RS attached DPP-IV, four hydrogen bonds are formed between RS and LG, and GLU205 of DPP-IV formed two hydrogen bonds, and SER630 formed one hydrogen bond with LG. There are two electrostatic bonds between LG and ARG125 of DDP- IV and TYR 547, PHE357, HIS740 of DDP-IV formed five hydrophobic bonds. Three intermolecular hydrophobic bonds between RS and LG are also formed. In the RS-PP complex, there are five hydrogen bonds in which GLU312, LEU306, and ARG880 of PP are involved. Two hydrophobic bonds between PHE909 and RS, two hydrophobic bonds between LYS905 and RS are also formed. There is also a pi-sulfur bond formed between TYR308 and RS. On the other hand when RS was docked against LG bounded PP two hydrogen bonds, one Pi-lone pair bond, eight hydrophobic bonds formed between RS and LG. GLU902, ARG886, TYR901, GLU312, TYR308, GLN119 of PP formed seven hydrogen bonds with RS, and PHE909 formed a hydrophobic bond with PP. A pi-sulfur bond was also present between TYR901 of PP and RS. 
Table 5 Non-covalent interactions of linagliptin (LG) and rabeprazole sodium (RS) with DPP-IV and proton pump enzyme.

\begin{tabular}{|c|c|c|c|c|c|c|}
\hline $\begin{array}{l}\text { Drug } \\
\text { Code }\end{array}$ & $\begin{array}{c}\text { Prote } \\
\text { in }\end{array}$ & $\begin{array}{c}\text { Binding } \\
\text { affinity } \\
\text { (kcal/mole) }\end{array}$ & Bond (Ligand and AA) & $\begin{array}{l}\text { Bond length } \\
(\AA)\end{array}$ & Bond type & Bond nature \\
\hline \multirow{9}{*}{ LG } & \multirow{9}{*}{$\begin{array}{l}\text { DPP } \\
\text {-IV }\end{array}$} & \multirow{9}{*}{-9.6} & A: SER630: HG - N: UNK1:O & 2.33258 & Hydrogen Bond & Conventional Hydrogen Bond \\
\hline & & & $\mathrm{N}:$ UNK1: H9 - N: UNK1: O & 2.44245 & Hydrogen Bond & Carbon Hydrogen Bond \\
\hline & & & N: UNK1: H9 - A: SER630: OG & 2.54809 & Hydrogen Bond & Carbon Hydrogen Bond \\
\hline & & & N: UNK1 - A: TYR547 & 4.49257 & Hydrophobic & Pi - Pi Stacked \\
\hline & & & A: TYR547 - N: UNK1 & 5.37978 & Hydrophobic & Pi-Alkyl \\
\hline & & & A: TRP629 - N: UNK1: C & 4.09495 & Hydrophobic & Pi-Alkyl \\
\hline & & & A: TRP629 - N: UNK1: C & 4.07497 & Hydrophobic & Pi-Alkyl \\
\hline & & & A: TYR662 - N: UNK1 & 5.17476 & Hydrophobic & Pi-Alkyl \\
\hline & & & A: TYR666 - N: UNK1 & 4.69523 & Hydrophobic & Pi-Alkyl \\
\hline \multirow{10}{*}{ RS } & \multirow{10}{*}{ PP } & \multirow{10}{*}{-6.5} & N: UNK1: HN - C: GLU312:OE1 & 2.94569 & Hydrogen Bond & Conventional Hydrogen Bond \\
\hline & & & N: UNK1: H9 - C: GLU312: OE1 & 2.33096 & Hydrogen Bond & Carbon Hydrogen Bond \\
\hline & & & N: UNK1: H17 - C: LEU306: O & 2.515 & Hydrogen Bond & Carbon Hydrogen Bond \\
\hline & & & N: UNK1: H21 - C: LEU306: O & 2.65983 & Hydrogen Bond & Carbon Hydrogen Bond \\
\hline & & & N: UNK1: H22 - C: ARG880: O & 2.59928 & Hydrogen Bond & Carbon Hydrogen Bond \\
\hline & & & N: UNK1: S - C: TYR308 & 5.29765 & Other & Pi-Sulfur \\
\hline & & & N: UNK1 - C: PHE909 & 5.6102 & Hydrophobic & Pi-Pi T-shaped \\
\hline & & & N: UNK1 - C: PHE909 & 4.91364 & Hydrophobic & Pi-Pi T-shaped \\
\hline & & & N: UNK1 - C: LYS905 & 5.28463 & Hydrophobic & Pi-Alkyl \\
\hline & & & N: UNK1 - C: LYS905 & 4.94951 & Hydrophobic & Pi-Alkyl \\
\hline \multirow{18}{*}{$\begin{array}{l}\text { LG- } \\
\text { (RS) }\end{array}$} & \multirow{18}{*}{$\begin{array}{l}\text { DPP } \\
\text {-IV }\end{array}$} & \multirow{18}{*}{-8.0} & N: UNK1: H - A: GLU205:O & 1.90065 & Hydrogen Bond & Conventional Hydrogen Bond \\
\hline & & & N: UNK1: HN - N: UNK1:O & 2.5938 & Hydrogen Bond & Conventional Hydrogen Bond \\
\hline & & & N: UNK1: HN - N: UNK1:O & 2.55948 & Hydrogen Bond & Conventional Hydrogen Bond \\
\hline & & & A: SER630: HG - N: UNK1:O & 2.19838 & Hydrogen Bond & Conventional Hydrogen Bond \\
\hline & & & N: UNK1: H10 - N: UNK1:O & 2.3601 & Hydrogen Bond & Carbon Hydrogen Bond \\
\hline & & & N: UNK1:H11 - A: GLU205:O & 2.6616 & Hydrogen Bond & Carbon Hydrogen Bond \\
\hline & & & A: HIS740:HD2 - N: UNK1:O & 3.01305 & Hydrogen Bond & Carbon Hydrogen Bond \\
\hline & & & N: UNK1:H17 - N: UNK1: N & 2.92884 & Hydrogen Bond & Carbon Hydrogen Bond \\
\hline & & & A: ARG125:NH2 - N: UNK1 & 3.60689 & Electrostatic & Pi-Cation \\
\hline & & & A: ARG125:NH2 - N: UNK1 & 3.81957 & Electrostatic & Pi-Cation \\
\hline & & & N: UNK1 - A: TYR547 & 5.01932 & Hydrophobic & Pi-Pi T-shaped \\
\hline & & & N: UNK1 - N: UNK1:C & 5.36156 & Hydrophobic & Pi-Alkyl \\
\hline & & & A: PHE357 - N: UNK1:C & 4.63111 & Hydrophobic & Pi-Alkyl \\
\hline & & & A: PHE357 - N: UNK1 & 4.53255 & Hydrophobic & Pi-Alkyl \\
\hline & & & A: TYR547 - N: UNK1 & 4.62434 & Hydrophobic & Pi-Alkyl \\
\hline & & & A: HIS740 - N: UNK1:C & 4.26833 & Hydrophobic & Pi-Alkyl \\
\hline & & & N: UNK1 - N: UNK1 & 4.90681 & Hydrophobic & Pi-Alkyl \\
\hline & & & N: UNK1 - N: UNK1:C & 3.95803 & Hydrophobic & Pi-Alkyl \\
\hline \multirow{21}{*}{$\begin{array}{l}\text { RS- } \\
\text { (LG) }\end{array}$} & \multirow{21}{*}{ PP } & \multirow{21}{*}{6.3} & N: UNK1: HN - N: UNK1: O & 2.42917 & Hydrogen Bond & Conventional Hydrogen Bond \\
\hline & & & N: UNK1: HN - A: GLU902: OE1 & 2.22923 & Hydrogen Bond & Conventional Hydrogen Bond \\
\hline & & & A: ARG886: HE - N: UNK1: O & 2.8135 & Hydrogen Bond & Conventional Hydrogen Bond \\
\hline & & & A: ARG886: HH22 - N: UNK1: O & 2.91449 & Hydrogen Bond & Conventional Hydrogen Bond \\
\hline & & & A: TYR901: HH - N: UNK1: O & 2.31814 & Hydrogen Bond & Conventional Hydrogen Bond \\
\hline & & & N: UNK1: H9 - A: GLU312: OE2 & 2.77391 & Hydrogen Bond & Carbon Hydrogen Bond \\
\hline & & & N: UNK1: H17 - A: TYR308: OH & 2.56535 & Hydrogen Bond & Carbon Hydrogen Bond \\
\hline & & & N: UNK1:H13 - N: UNK1: O & 1.90708 & Hydrogen Bond & Carbon Hydrogen Bond \\
\hline & & & A: GLN119:HE21 - N: UNK1 & 3.07905 & Hydrogen Bond & Pi-Donor Hydrogen Bond \\
\hline & & & N: UNK1:S - A: TYR901 & 5.24684 & Other & Pi-Sulfur \\
\hline & & & N: UNK1:O - N: UNK1 & 2.87092 & Other & Pi-Lone Pair \\
\hline & & & N: UNK1 - N: UNK1 & 5.8873 & Hydrophobic & Pi-Pi T-shaped \\
\hline & & & N: UNK1 - N: UNK1 & 5.52328 & Hydrophobic & Pi-Pi T-shaped \\
\hline & & & N: UNK1:C - N: UNK1: C & 3.66064 & Hydrophobic & Alkyl \\
\hline & & & $\mathrm{N}$ : UNK1:C - N: UNK1 & 3.4677 & Hydrophobic & Alkyl \\
\hline & & & N: UNK1 - N: UNK1: C & 5.36794 & Hydrophobic & Pi-Alkyl \\
\hline & & & $\mathrm{N}:$ UNK1 - N: UNK1 & 5.10605 & Hydrophobic & Pi-Alkyl \\
\hline & & & N: UNK1 - N: UNK1: C & 4.81474 & Hydrophobic & Pi-Alkyl \\
\hline & & & N: UNK1 - A: PRO118 & 4.87555 & Hydrophobic & Pi-Alkyl \\
\hline & & & N: UNK1 - N: UNK1: C & 5.06755 & Hydrophobic & Pi-Alkyl \\
\hline & & & A: PHE909 - N: UNK1: C & 4.95947 & Hydrophobic & Pi-Alkyl \\
\hline
\end{tabular}

Note LG = Linagliptin, RS = Rabeprazole sodium, DPP-IV = Dipeptidyl peptidase IV, PP = proton pump, AA = Amino acid, ARG = Arginine, $\mathrm{PRO}=$ Proline, $\mathrm{LEU}=$ Leucine, $\mathrm{GLU}=$ Glutamate, $\mathrm{SER}=$ Serine, $\mathrm{PHE}=$ Phenylalanine, $\mathrm{TYR}=$ Tyrosine, $\mathrm{TRP}=$ Tryptophan, HIS $=$ Histidine . 
(A)

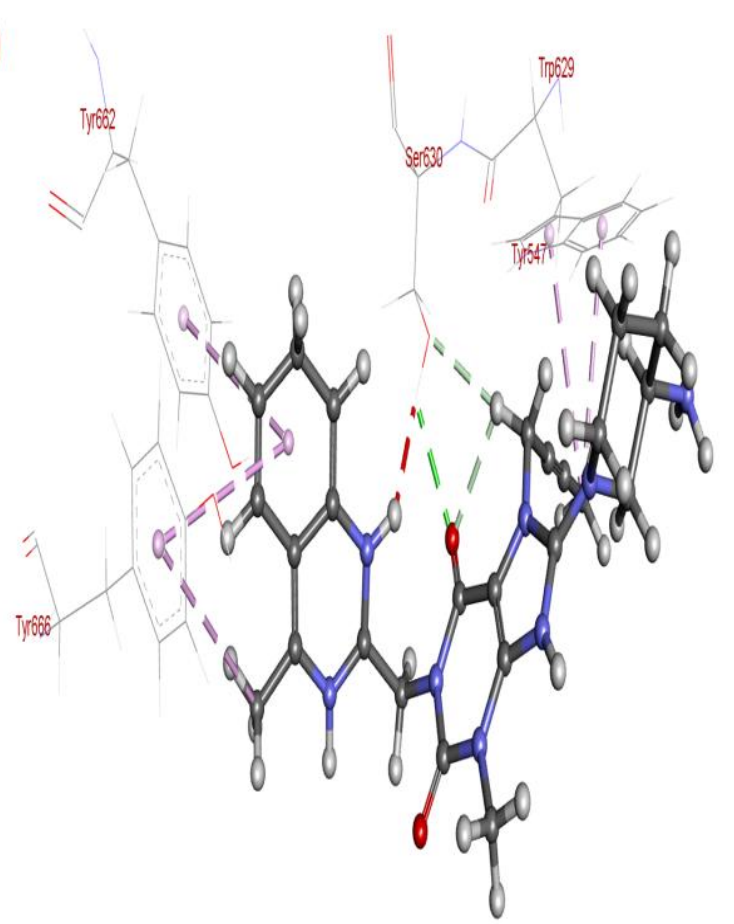

(B)

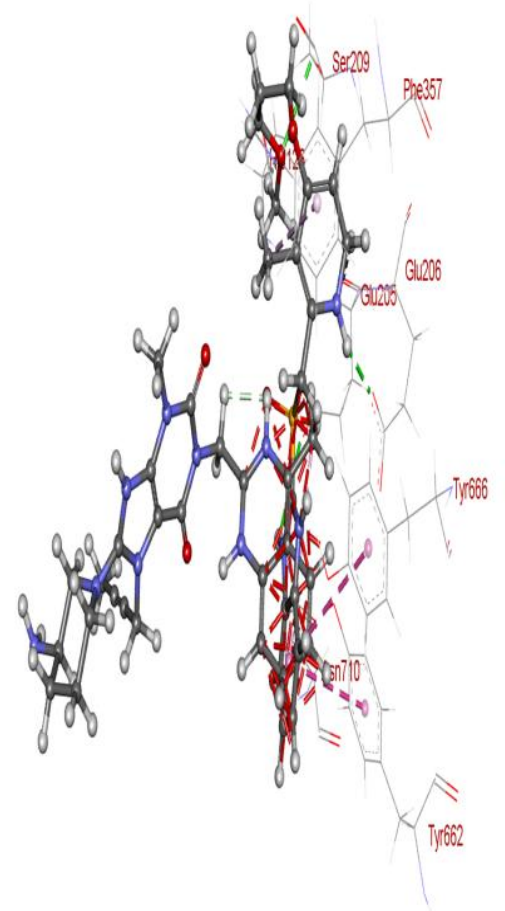

(C)

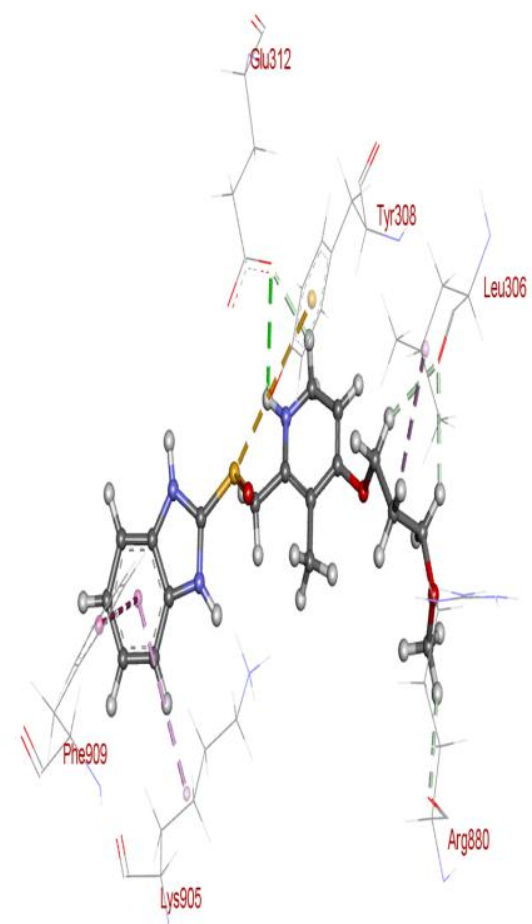

(D)

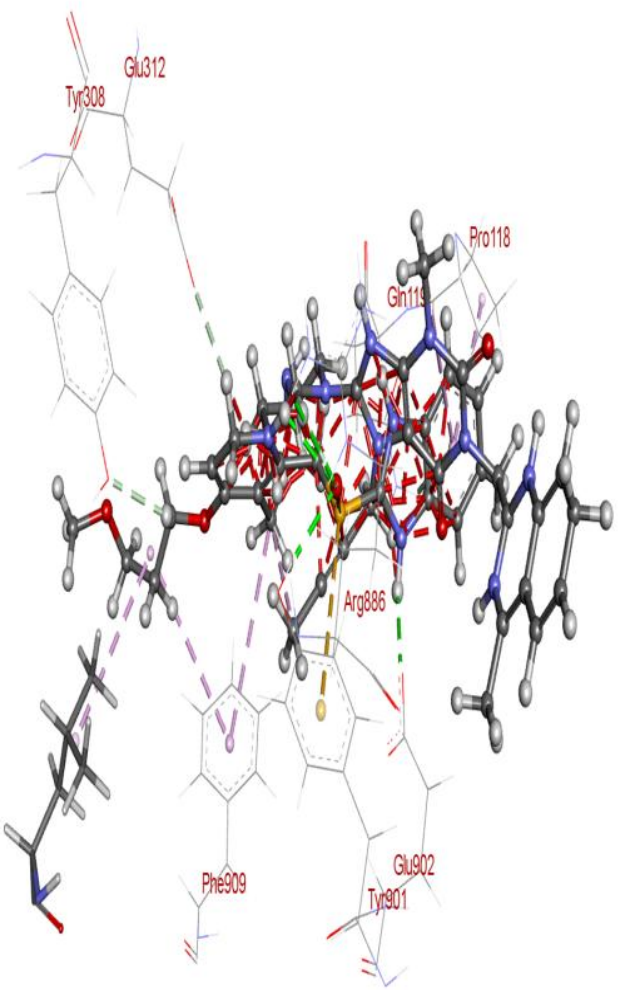

Figure 5 Molecular docking models (3D) of (A) LG-DPP-IV, (B) LG-(RS-DPP-IV), (C) RS-PP, and (D) RS-(LG-PP). [Here, $\mathrm{LG}=$ linagliptin, $\mathrm{RS}=$ rabeprazole sodium] . 

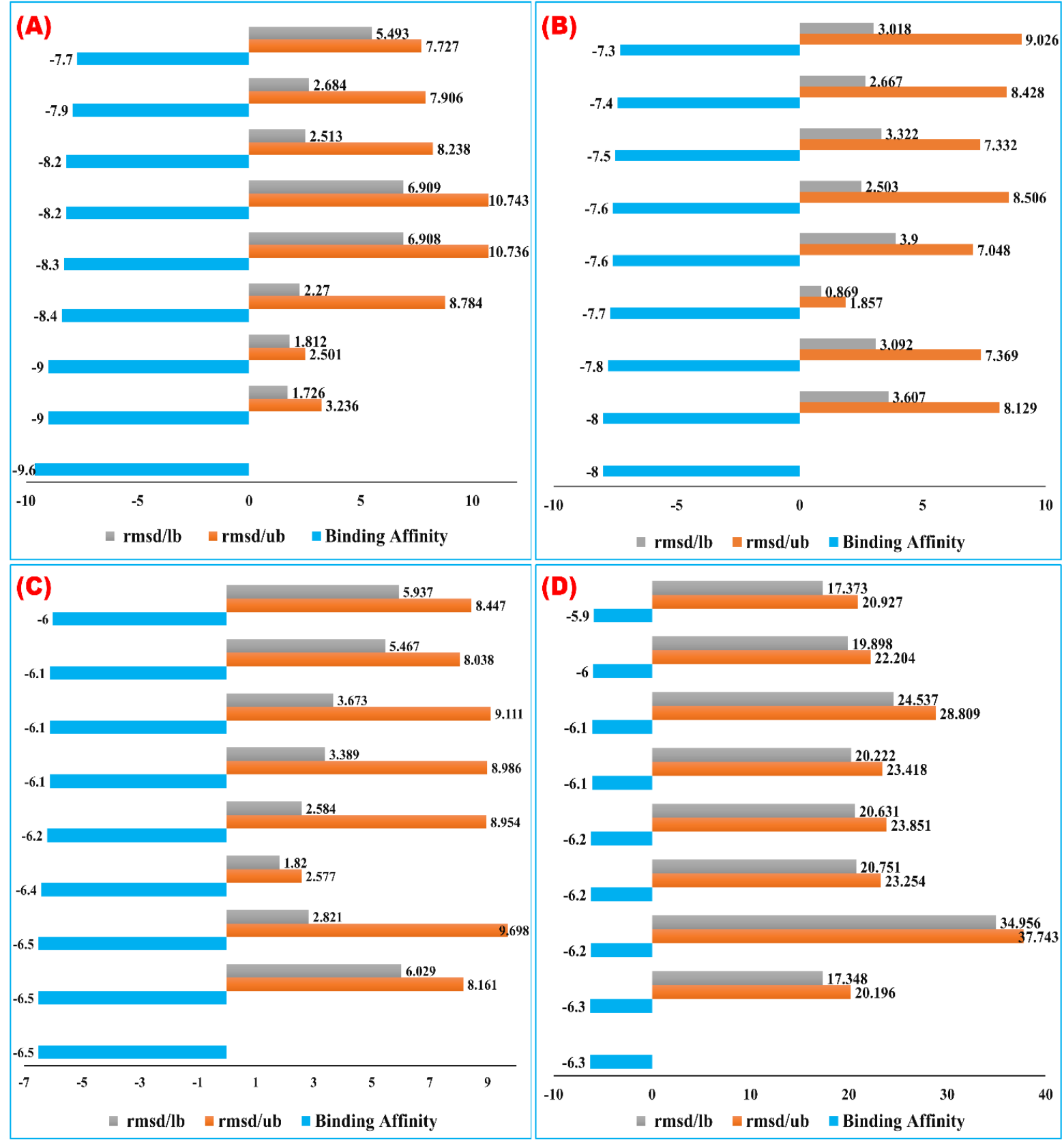

Figure 6 Binding affinity (kcal/mole) values of (A) LG, and (B) LG-RS with DPP-IV, and (C) RS, and LG-RS with proton pump. [Here, $\mathrm{LG}=$ linagliptin, $\mathrm{RS}=$ rabeprazole sodium $]$.

\section{Discussion}

This study attempted to discover whether Rabeprazole Sodium and Linagliptin interact with each other in-vitro and in-silico if administered simultaneously. The thermal analyses which comprised melting point study and thermogravimetric analysis demonstrated that these two drug molecules showed different physical characteristics upon complexation. TGA data also showed that the LG-RS complex underwent different degradation patterns than the individual drugs which solidified the notion that these drugs did interact with each other. It was further displayed with the SEM and XRPD analyses data. XRPD data clearly showed that the LG-RS complex was of less crystalline characteristics than LG alone. The particle size of the complex was also found to be reduced than that of LG. A similar conclusion was also achieved after the SEM data. It further showed the topographical changes of 
the complex and the differences between the complex and the individual drugs. These changes in physical features might result in a significant alteration in the therapeutic activity of the antidiabetic or anti-acidity drugs (Shariare et al., 2011; Chuah et al., 2014).

Possible mechanisms of interaction were explored via the synchronous fluorescence spectroscopic analysis and molecular docking analysis. From these data, it was evident that the LG-RSBSA system showed significantly reduced Stern-Volmer quenching constant and quenching rate constant than those of both LG-BSA and RS-BSA systems, and therefore might show weaker interaction with the BSA protein. It was found that protein binding features were different in the case of the complex than that of LG. From the quenching rate constant values, it may be said that the quenching mechanism might not have been altered, i.e., the complex still followed the static quenching mechanism (Rajbangshi et al., 2018). All in all, these data demonstrated that the LG-RS complex showed altered protein binding than LG, and these alterations might interfere with the efficacy and safety profile of the drug, as described in previous studies (Ojha \& Das, 2011; Shen et al., 2015). The molecular docking analysis of these two parent drugs and their complex demonstrated the binding profile of these drugs with their respective target receptors. The binding affinity has reduced substantially due to the complexation between the drugs. All these docking results are supported by the results obtained from synchronous fluorescence spectroscopic methods. Besides, all the in vitro and in-silico findings were supported by several previous studies (Hossain et al., 2020c, 2021). All in all, these two drugs might show drug-drug interactions together resulting in altered efficacy and safety of the individual drugs (Ojha \& Das, 2011; Shen et al., 2015). Further in vivo studies and extensive human data monitoring are essential to determine whether this interaction is significant clinically.

\section{Conclusions}

Drug interaction has become a serious issue worldwide as multiple drugs are often prescribed simultaneously. Linagliptin and rabeprazole sodium are drugs of two different classes but are often prescribed together. However, noteworthy drug-drug interaction has been observed between these two moieties. Thermal analysis, including melting point study and thermogravimetric analytical data, of the 1:1 mixture of these two drugs, clearly demonstrated that these compounds form a complex of different physical characteristics. The XRPD and SEM analysis also supported this finding. These data showed that, upon the mixing of linagliptin and rabeprazole sodium, the morphological features of the mixed product do not match with those of the initial drugs. The crystalline characteristics were found to be modified and the mean particle size and particle size distribution were changed. The molecular docking data and the synchronous fluorescence spectroscopic analysis including the change in Stern-Volmer plots and Stern-
Volmer constants illustrated how these interactions might have occurred. Besides, it also showed how the plasma protein binding of the drugs might have been altered and which amino acid residue might be responsible for this. The efficacy of any drug depends on its pharmacokinetic and pharmacodynamic parameters. Physical interaction between multiple drugs may alter the absorption, distribution, metabolism, and excretion features of said drugs and may result in increased or decreased therapeutic activity. As a result, dose correction may be necessary. Besides, drug interactions may introduce newer adverse effects and may render the drugs unsafe for use. The findings of this study strongly indicate that physical drug interaction may occur between linagliptin and rabeprazole sodium when these drugs are taken simultaneously. These findings also predict that the biological activity of the drugs may also be changed due to these interactions. Further studies are necessary to verify these effects in humans and establish whether the interaction is significant therapeutically.

\section{Acknowledgements}

All the authors of the manuscript profoundly acknowledge to the Department of Pharmaceutical Chemistry, and Centre for Advanced Research in Sciences (CARS), University of Dhaka, Dhaka, Bangladesh for the lab and logistical support.

\section{Author's contributions}

This exertion was employed in teamwork of all authors. All the authors have accepted responsibility for the entire content of this submitted manuscript and approved the submission. MJH was involved in conception and design of the experiments. MJH, MSI, Saimon Shahriar (SS), Sherejad Sanam (SS), and SM contributed to perform the experiments. MJH, Sherejad Sanam (SS), MSI, CSK, MRI, and TBE analyzed data. MJH, MSI, Saimon Shahriar (SS), CSK, and SM authors contributed to drafting the article. TBE, MRI, and KD contributed to revising it critically for important intellectual content. All authors made the final approval of the version to be published.

\section{Conflict of interest}

The authors declare no conflict of interest

\section{References}

Abbas Moussa B, Mahrouse MA, Fawzy MG (2019) A validated LC-MS/MS method for simultaneous determination of linagliptin and metformin in spiked human plasma coupled with solid phase extraction: Application to a pharmacokinetic study in healthy volunteers. Journal of Pharmaceutical and Biomedical Analysis 163:153-161.

Ahmed S, Islam N, Shahinozzaman M, Fakayode SO, Afrin N, et al. (2021) Virtual screening, molecular dynamics, density 
functional theory and quantitative structure activity relationship studies to design peroxisome proliferator-activated receptor- $\gamma$ agonists as anti-diabetic drugs. Journal of Biomolecular Structure and Dynamics 39(2):728-742.

Ambike AA, Mahadik KR, Paradkar A (2005) Spray-dried amorphous solid dispersions of simvastatin, a low tg drug: in-vitro and in-vivo evaluations. Pharmaceutical Research 22(6):990-998.

Arab HH, Eid AH, Mahmoud AM, Senousy MA (2021) Linagliptin mitigates experimental inflammatory bowel disease in rats by targeting inflammatory and redox signaling. Life Sciences 273:119295.

Barden AT, de Abreu Engel RE, Campanharo SC, Volpato NM, Schapoval EE (2017) Characterization of linagliptin using analytical techniques. Drug Analytical Research 1(2):30-37.

Ceriello A, Inagaki N (2017) Pharmacokinetic and pharmacodynamic evaluation of linagliptin for the treatment of type 2 diabetes mellitus, with consideration of Asian patient populations. Journal of Diabetes Investigation 8(1):19-28.

Chuah AM, Jacob B, Jie Z, Ramesh S, Mandal S, et al. (2014) Enhanced bioavailability and bioefficacy of an amorphous solid dispersion of curcumin. Food Chemistry 156:227-233.

Dash RP, Rais R, Srinivas NR (2018) Stereoselective and nonstereoselective pharmacokinetics of rabeprazole - an overview. Xenobiotica 48(4):422-432.

Hossain MJ, Rashid MA, Sultan MZ (2020a) Transition metal chelation augments the half-life of secnidazole: molecular docking and fluorescence spectroscopic approaches. Drug Research 70(12):583-592.

Hossain MJ, Sultan MZ, Rashid MA, Kuddus MR (2020b) In-vitro interactions of secnidazole and its iron (ii), copper (ii) complexes with bovine serum albumin by fluorescence quenching method. Bangladesh Pharmaceutical Journal 23(1):1-9.

Hossain MJ, Sultan MZ, Rashid MA, Kuddus MR (2020c) Does rabeprazole sodium alleviate the anti-diabetic activity of linagliptin? Drug-drug interaction analysis by in-vitro and in-vivo methods. Drug Research 70(11):519-27.

Hossain MJ, Sultan MZ, Rashid MA, Kuddus MR (2021) Interactions of linagliptin, rabeprazole sodium, and their formed complex with bovine serum albumin: Computational docking and fluorescence spectroscopic methods. Analytical Science Advances $1-15$.

Huang Y, Lu H, Zhang F, Min C (2018) Identification, isolation, characterization, and UHPLC quantification of potential genotoxic impurities in linagliptin. Journal of Separation Science 41(21):3985-3994.

Kang WY, Lee HW, Gwon M-R, Cho S, Shim W-S, et al. (2020) A pharmacokinetic drug interaction between fimasartan and linagliptin in healthy volunteers. Drug Design, Development and Therapy 14:2101-2111.

Li G, Liu BS, Zhang Q, Han R (2016) Investigation on the effect of fluorescence quenching of bovine serum albumin by cefoxitin sodium using fluorescence spectroscopy and synchronous fluorescence spectroscopy. Luminescence 31(5):1054-1062.

Ochoa D, Román M, Cabaleiro T, Saiz-Rodríguez M, Mejía G, et al. (2020) Effect of food on the pharmacokinetics of omeprazole, pantoprazole and rabeprazole. BMC Pharmacology and Toxicology 21(1):54.

Ojha B, Das G (2011) Role of hydrophobic and polar interactions for BSA-amphiphile composites. Chemistry and Physics of Lipids 164(2):144-50.

Patel BH, Madhabhai M. Patel, Patel JR, Suhagia BN (2007) HPLC analysis for simultaneous determination of rabeprazole and domperidone in pharmaceutical formulation. Journal of Liquid Chromatography \& Related Technologies 30(3):439-445.

Patel P, Leeder JS, Piquette-Miller M, Dupuis LL (2017) Aprepitant and fosaprepitant drug interactions: a systematic review. British Journal of Clinical Pharmacology 83(10):2148-2162.

Rajbangshi JC, Alam MM, Hossain MS, Islam MS, Rouf ASS (2018) Development and validation of a RP-HPLC method for quantitative analysis of linagliptin in bulk and dosage forms. Dhaka University Journal of Pharmaceutical Sciences 17(2):175-182.

Rosenstock J, Perkovic V, Johansen OE, Cooper ME, Kahn SE, et al. (2019) Effect of linagliptin vs placebo on major cardiovascular events in adults with type 2 diabetes and high cardiovascular and renal risk: the CARMELINA randomized clinical trial. Journal of American Medical Association 321(1):69-79.

Sanad MH, Challan SB (2017) Radioiodination and biological evaluation of rabeprazole as a peptic ulcer localization radiotracer. Radiochemistry 59(3):307-312.

Shariare MH, de Matas M, York P, Shao Q (2011) The impact of material attributes and process parameters on the micronisation of lactose monohydrate. International Journal of Pharmaceutics 408(1):58-66.

Shen GF, Liu TT, Wang Q, Jiang M, Shi JH (2015) Spectroscopic and molecular docking studies of binding interaction of gefitinib, 
lapatinib and sunitinib with bovine serum albumin (BSA). Journal of Photochemistry and Photobiology B 153:380-390.

Suryawanshi VD, Walekar LS, Gore AH, Anbhule PV, Kolekar GB (2016) Spectroscopic analysis on the binding interaction of biologically active pyrimidine derivative with bovine serum albumin. Journal of Pharmaceutical Analysis 6(1):56-63.

Tannenbaum C, Sheehan NL (2014) Understanding and preventing drug-drug and drug-gene interactions. Expert Review of Clinical Pharmacology 7(4):533-544.

Trott O, Olson AJ (2010) AutoDock Vina: improving the speed and accuracy of docking with a new scoring function, efficient optimization, and multithreading. Journal of Computational Chemistry 31(2):455-61.

Ullah Z, Atiq S, Naseem S (2013) Indexing the diffraction patterns and investigating the crystal structure of pb-doped strontium ferrites. Journal of Scientific Research 5(2),235-244.
Wang BL, Pan DQ, Zhou KL, Lou YY, Shi JH (2019) Multispectroscopic approaches and molecular simulation research of the intermolecular interaction between the angiotensin-converting enzyme inhibitor (ACE inhibitor) benazepril and bovine serum albumin (BSA). Spectrochimica Acta Part A: Molecular and Biomolecular Spectroscopy 212:15-24.

Weinstein ZB, Kuru N, Kiriakov S, Palmer AC, Khalil AS, et al. (2018) Modeling the impact of drug interactions on therapeutic selectivity. Nature Communications 9(1):1-9.

Weinstein ZB, Zaman MH (2017) Quantitative bioassay to identify antimicrobial drugs through drug interaction fingerprint analysis. Scientific Reports 7(1):1-8.

Zhang YF, Zhou KL, Lou YY, Pan DQ, Shi JH (2017) Investigation of the binding interaction between estazolam and bovine serum albumin: multispectroscopic methods and molecular docking technique. Journal of Biomolecular Structure and Dynamics 35:3605-3614. 